International Journal of Pure and Applied Mathematics

Volume 84 No. 3 2013, 139-152

ISSN: 1311-8080 (printed version); ISSN: 1314-3395 (on-line version)

url: http://www.ijpam.eu

doi: http://dx.doi.org/10.12732/ijpam.v84i3.1

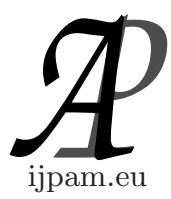

\title{
MINIMAL TWO-VALUED ECCENTRIC SEQUENCES
}

\author{
P. Hrnčiar ${ }^{1 ~}$, G. Monoszová ${ }^{2}$ \\ ${ }^{1,2}$ Department of Mathematics \\ Faculty of Natural Sciences \\ Matej Bel University \\ Tajovského 40, 97401 Banská Bystrica, SLOVAKIA
}

\begin{abstract}
An eccentric sequence of a connected finite graph is a sequence of eccentricities of its vertices. An eccentric sequence is called minimal if it has no proper eccentric subsequence with the same number of distinct eccentricities. We survey known results concerning minimal two-valued eccentric sequences and describe a new infinite class of these sequences. Also a conjecture on all minimal two-valued eccentric sequences is proposed.
\end{abstract}

AMS Subject Classification: $05 \mathrm{C} 12$

Key Words: cycle, eccentricity, eccentric sequence, minimal eccentric sequence

\section{Introduction}

One concept that pervades all of graph theory is that of distance. Among important notions defined through distance there is the eccentricity of a vertex of a connected graph. The list of the eccentricities (in nondecreasing order) of the vertices of a connected finite graph $G$ is the eccentric sequence of $G$. An eccentric sequence is called minimal if it has no proper eccentric subsequence with the same number of distinct eccentricities. Characterization of eccentric sequences is considered to be an important problem in graph theory (see Problem 1 in [3]). This problem is extremely difficult. It seems that even finding all

Received: December 14, 2011

(c) 2013 Academic Publications, Ltd.

$\S_{\text {Correspondence author }}$ url: www.acadpubl.eu 
minimal eccentric sequences with least eccentricity five is a very difficult problem. Eccentric sequences have an important role in aplication of graph theory (see, for instance, [4], [9] and [13]). It is not excluded that eccentric sequences could have a distinguished role in describing reality on the fundamental level.

A survey of known results relating to the topic of the paper is given in its second section. The main results are given in the third section. In that section a new infinite class of minimal eccentric sequences with two values is described and also a new proof for known sequences of this type is given. A conjecture at the end of the third section completes a description of all minimal two-valued eccentric sequences. Since the proof of Theorem 3.2 is rather complicated, proofs of several needed lemmas are presented in the last section of the paper.

For a connected graph $G$, the distance $d_{G}(u, v)$ or briefly $d(u, v)$ between vertices $u$ and $v$ is the length of a shortest path joining them. The distance between a vertex $u \in V(G)$ and a subgraph $H$ of $G$ will be denoted by $d_{G}(u, H)$, i.e. $d_{G}(u, H)=\min \left\{d_{G}(u, v) ; v \in V(H)\right\}$. The eccentricity $e_{G}(u)$ (briefly $e(u)$ ) of a vertex $u \in V(G)$ is the distance to a vertex farthest from $u$ in $G$, i.e. $e_{G}(u)=\max \left\{d_{G}(u, v) ; v \in V(G)\right\}$. The radius $\operatorname{rad} G$ of $G$ is the minimum eccentricity among the vertices of $G$ while the diameter $\operatorname{diam} G$ of $G$ is the maximum eccentricity. The eccentricity sequence es $(G)$ of $G$ is the list of the eccentricities of its vertices in nondecreasing order. Since there are often many vertices having the same ecentricity, we will simplify the sequence by listing it as $e_{1}^{m_{1}}, e_{2}^{m_{2}}, \ldots, e_{k}^{m_{k}}$, i.e. es $(G)=\left(e_{1}^{m_{1}}, e_{2}^{m_{2}}, \ldots, e_{k}^{m_{k}}\right)$. An eccentric sequence is called minimal if it has no proper eccentric subsequence with the same number of distinct eccentricities ([11]). Let $C$ be a cycle of $G$. A vertex $u$ of the cycle $C$ is called $C$-excited (in $G$ ) if $e_{G}(u)>e_{C}(u)$. The number of C-excited vertices is denoted by $\operatorname{exc}_{G} C$, i.e. $\operatorname{exc}_{G} C=\left|\left\{u \in V(C) ; e_{G}(u)>e_{C}(u)\right\}\right|$ (see [7]). A cycle $C$ in $G$ is called geodesic if for any two vertices of $C$ their distance in $C$ equals their distance in $G$. The circumference $c(G)$ of $G$ is the length of any longest cycle in $G$. A connected unicyclic graph $G$ with the cycle $C$ is called a sun-graph (see [7]) if $\operatorname{deg}_{G}(u) \leq 3$ for $u \in V(C)$ and $\operatorname{deg}_{G}(u) \leq 2$ for $u \in V(G) \backslash V(C)$. A $u-v$ path $P$ in a sun-graph $G$ is called a ray if $V(P) \cap V(C)=\{u\}$ and $\operatorname{deg}_{G}(v)=1$.

\section{Survey}

In this section we recall the known results relating to the topic of this paper (see also [1], [2] and [5]).

Theorem 2.1. (see [10]) 
a) A sequence of positive integers is eccentric if and only if some subsequence of it with the same number of distinct members is eccentric.

b) If $\operatorname{es}(G)=\left(e_{1}^{m_{1}}, e_{2}^{m_{2}}, \ldots, e_{k}^{m_{k}}\right)$ and $k \geq 2$ then $e_{i}=e_{i-1}+1$ and $m_{i} \geq 2$ for all $i \in\{2,3, \ldots, k\}$.

Theorem 2.2. [7] Let $G$ be a graph with $\operatorname{rad} G=r, \operatorname{diam} G \leq 2 r-2$, on at most $3 r-2$ vertices. Then $G$ contains a geodesic cycle of length $2 r$ or $2 r+1$.

Lemma 2.3. [7] Let $C$ be a cycle of a connected graph $G$ and $\mid V(G) \backslash$ $V(C) \mid=k$. Then

a) $\operatorname{exc}_{G}(C) \leq 2 k-1$ if the length of $C$ is even and $k \geq 1$,

b) $\operatorname{exc}_{G}(C) \leq 2 k$ if the length of $C$ is odd,

c) $\operatorname{exc}_{G}(C) \leq 2 k-l$ if the length of $C$ is even and $l=\left|\left\{u \in V(G) ; d_{G}(u, C)=1\right\}\right|$.

Theorem 2.4. [8] Let $r \geq 3$ and $G$ be a graph with es $(G)=\left(r^{\alpha},(r+1)^{\beta}\right)$. Then

a) there exists a block $B$ of $G$ which contains all cut-vertices of $G$ and moreover with the property that for every $u \in V(G) \backslash V(B)$ it holds $d_{G}(u, B)=1$,

b) for circumference of $G$ and for the block $B$ from the previous it holds $c(G) \geq c(B) \geq 2 r-2$,

c) if $c(G)<2 r$ then $\alpha \geq 2 r-2$.

Theorem 2.5. [11] The minimal eccentric sequences with least eccentricity at most two are precisely: $0 ; 1^{2} ; 1,2^{2} ; 2^{4} ; 2^{2}, 3^{2} ; 2,3^{6} ; 2,3^{2}, 4^{2}$.

Theorem 2.6. [6] There are exactly 13 minimal eccentric sequences with least eccentricity three, namely: $3^{6} ; 3^{5}, 4^{2} ; 3^{4}, 4^{4} ; 3^{3}, 4^{6} ; 3^{2}, 4^{8} ; 3,4^{10}$; $3,4^{2}, 5^{12} ; 3,4^{3}, 5^{9} ; 3,4^{4}, 5^{7} ; 3,4^{5}, 5^{4} ; 3,4^{7}, 5^{2} ; 3^{2}, 4^{2}, 5^{2} ; 3,4^{2}, 5^{2}, 6^{2}$.

Theorem 2.7. [8] There are exactly 7 minimal eccentric sequences of type $4^{\alpha}, 5^{\beta}$, namely: $4^{7}, 5^{2} ; 4^{6}, 5^{4} ; 4^{5}, 5^{6} ; 4^{4}, 5^{8} ; 4^{3}, 5^{9} ; 4^{2}, 5^{12} ; 4,5^{14}$.

From the last three theorems it is evident that all minimal two-valued eccentric sequences with less eccentricity at most four are known. The following theorem describes minimal two-valued eccentric sequences with less eccentricity $r \geq 5$. 
Theorem 2.8. [7]

a) The sequences $5^{9}, 6^{2} ; 5^{8}, 6^{4} ; 5^{7}, 6^{6} ; 5^{6}, 6^{8} ; 5^{5}, 6^{9}$ are minimal eccentric sequences.

b) Al minimal eccentric sequences of type $r^{\alpha},(r+1)^{\beta}$ for $r \geq 6$ and $\alpha+\beta \leq$ $\frac{8 r+5}{3}$ are:

$$
\begin{aligned}
& r^{2 r-i},(r+1)^{2 i}, i=1,2, \\
& r^{2 r-2 i+1},(r+1)^{3 i}, i=2,3, \ldots,\left\lfloor\frac{2 r+1}{3}\right\rfloor, \\
& r^{2 r-2 i},(r+1)^{3 i+2}, i=2,3, \ldots,\left\lfloor\frac{2 r-1}{3}\right\rfloor .
\end{aligned}
$$

Note that all minimal one-valued eccentric sequences are also known. These sequences are 0 and $r^{2 r}$ for $r \geq 1$ (see[12],[7]).

\section{Main Results}

The following lemma is a generalization of Lemma 2.3.

Lemma 3.1. Let $G$ be a connected graph which contains a cycle $C$ and $G$ ' be a unicyclic connected subgraph of $G$ with the cycle $C$. Then

$$
\operatorname{exc}_{G}(C) \leq \operatorname{exc}_{G^{\prime}}(C)+2 .\left(|V(G)|-\left|V\left(G^{\prime}\right)\right|\right) .
$$

Proof. Let $k=|V(G)|-\left|V\left(G^{\prime}\right)\right|$. Consider a sequence $G_{0}=G^{\prime}, G_{1}, \ldots$, $G_{k}$ of subgraphs of $G$ such that $G_{i+1}=G_{i}+u v(i<k)$, where $u \in V\left(G_{i}\right)$, $v \in V\left(G_{i+1}\right) \backslash V(G), u v \in E(G)$. Obviously, $\operatorname{exc}_{G_{i+1}}(C) \leq \operatorname{exc}_{G_{i}}(C)+2$. Since $\operatorname{exc}_{G}(C) \leq \operatorname{exc}_{G_{k}}(C)$, we get $\operatorname{exc}_{G}(C) \leq \operatorname{exc}_{G^{\prime}}(C)+2 k=\operatorname{exc}_{G^{\prime}}(C)+2(|V(G)|-$ $\left.\left|V\left(G^{\prime}\right)\right|\right)$.

It is easy to see (it is sufficient to consider a suitable subgraph $G^{\prime}$ of $G$ ) that the cases a), b), c) in Lemma 2.3 are consequences of Lemma 3.1.

Minimal eccentric sequences from the case (i) of the following theorem are known, except of the sequence $5^{4}, 6^{11}$, since 2004 (see Theorem 2.8). However, the assumptions in Theorem 3.2 are formulated more transparently than in Theorem 2.8 and we also give a new proof that the eccentric sequences from the case (i) are minimal.

Theorem 3.2. All minimal eccentric sequences of type $r^{\alpha},(r+1)^{\beta}$ for $r \geq 5$ and $\alpha \geq \frac{r+3}{2}$ are:

(i) $r^{2 r-i},(r+1)^{2 i}, i=1,2$,

$r^{2 r-2 i+1},(r+1)^{3 i}, i=2,3, \ldots,\left\lfloor\frac{2 r+1}{3}\right\rfloor$,
$r^{2 r-2 i},(r+1)^{3 i+2}, i=2,3, \ldots,\left\lfloor\frac{2 r-1}{3}\right\rfloor$, 


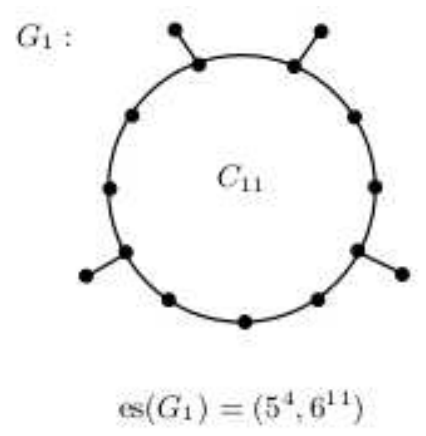

Figure 1

(ii) $r^{\alpha},(r+1)^{4 r-3 \alpha+2}$, if $r=9$ or $r \geq 11$, and $\frac{r+3}{2} \leq \alpha \leq \frac{2 r}{3}$.

Proof. a) First we show that each of the given sequences is eccentric.

A sequence $r^{2 r-i},(r+1)^{2 i}$ for $i=1,2$ is realizable through a sun-graph with the cycle $C_{2 r}$ of length $2 r$ and with $i$ rays of length one. A sequence $r^{2 r-2 i+1},(r+1)^{3 i}$ for $i=2,3, \ldots,\left\lfloor\frac{2 r+1}{3}\right\rfloor$ is realizable through any sun-graph with the cycle $C_{2 r+1}$, with $i$ rays of length one and such that $\operatorname{exc}_{G} C_{2 r+1}=2 i$ and no $C_{2 r+1}$-excited vertex is a cut-vertex of $G$ (we recommend to see Figures 3.11 - 3.13 in [7]). Analogously, a sequence $r^{2 r-2 i},(r+1)^{3 i+2}$ for $i=2,3, \ldots,\left\lfloor\frac{2 r-1}{3}\right\rfloor$ is realizable through any sun-graph $G$ with the cycle $C_{2 r+1}$, with $i+1$ rays of length one and such that $\operatorname{exc}_{G} C_{2 r+1}=2 i+1$ and no $C_{2 r+1}$-excited vertex is a cut-vertex of $G$ (see Figures $3.15-3.17$ in [7]). For instance the sequence $5^{4}, 6^{11}$ is realizable through the graph $G_{1}$ in Figure 1 .

A sequence of type $r^{\alpha},(r+1)^{4 r-3 \alpha+2}$ is realizable through a graph $G$, which can be constructed in two steps as follows:

$\left(1^{\circ}\right)$ We construct a sun-graph $G^{\prime}$ with the cycle $C_{2 r+1}$, with $\alpha$ rays of length one and such that $\operatorname{exc}_{G^{\prime}} C_{2 r+1}=2 \alpha$ and no $C_{2 r+1}$-excited vertex is a cut-vertex of $G^{\prime}$.

$\left(2^{\circ}\right)$ We add $2 r+1-3 \alpha$ new vertices and $2(2 r+1-3 \alpha)$ new edges to the graph $G^{\prime}$ by the following rule. If a vertex $u$ of $G^{\prime}$ is not a cut-vertex of $G^{\prime}, e_{G^{\prime}}(u)=r$ and $d_{G^{\prime}}\left(u, u_{1}\right)=d_{G^{\prime}}\left(u, u_{2}\right)=r$ for $u_{1}, u_{2} \in V\left(C_{2 r+1}\right)$, $u_{1} \neq u_{2}$, then we add a new vertex $u^{\prime}$ and two edges $u^{\prime} u_{1}, u^{\prime} u_{2}$. For example, Figure 2 shows the graph $G_{2}$ with eccentric sequence $21^{13}, 22^{47}$.

b) We show that each of the considered eccentric sequences is minimal.

The sequence $5^{4}, 6^{11}$ is minimal according to Lemma 4.1. In what follows we will suppose that $\alpha \neq 4$ for $r=5$. 


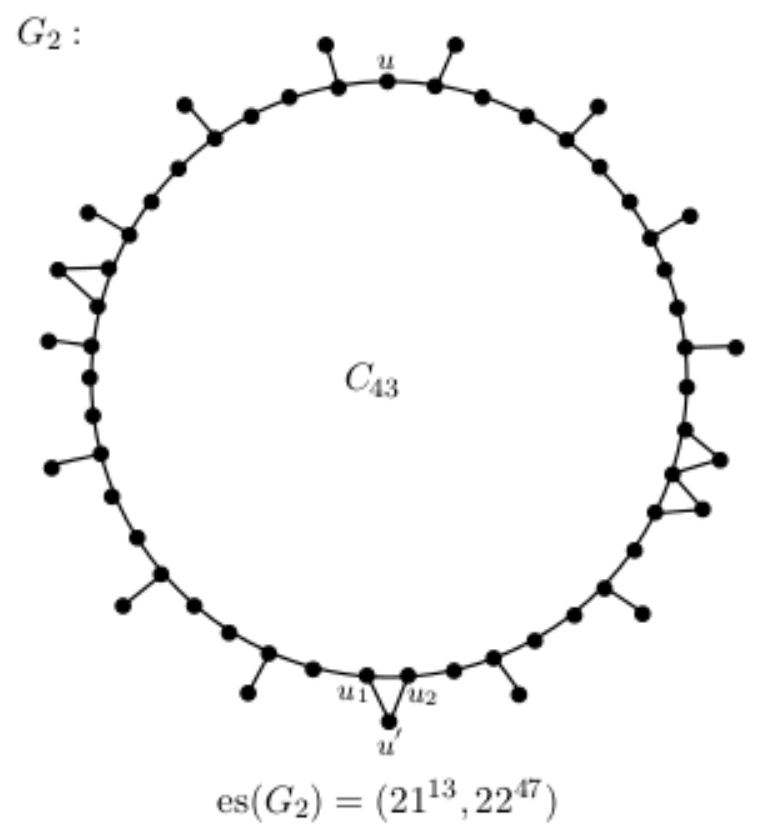

Figure 2

First we show that $\alpha+\beta \leq 3 r-1$ for each sequence $r^{\alpha},(r+1)^{\beta}$, except the sequence $5^{4}, 6^{11}$.

If a considered sequence is from the case (i), then it is easy to see that $\alpha+\beta \leq$ $2 r+\left\lfloor\frac{2 r-1}{3}\right\rfloor+2$. We get $\alpha+\beta \leq 2 r+\frac{2 r-1}{3}+2=\frac{8 r+5}{3}$ and $\frac{8 r+5}{3} \leq 3 r-1$ for $r \geq 8$. Further it is easy to verify that $\alpha+\beta \leq 3 r-1$ also for $r \in\{5,6,7\}$.

If a considered sequence is from the case (ii), then $\alpha \geq \frac{r+3}{2}$ and we get $\alpha+\beta=$ $4 r-2 \alpha+2 \leq 3 r-1$.

Now we are going to show that any considered sequence, except $5^{4}, 6^{11}$, is minimal. Suppose, contrary to our claim, that $r^{\alpha},(r+1)^{\beta}$ is not a minimal eccentric sequence. Then there exists a graph $G^{\prime}$ such that es $\left(G^{\prime}\right)=\left(r^{\alpha^{\prime}},(r+\right.$ $\left.1)^{\beta^{\prime}}\right), \alpha^{\prime}+\beta^{\prime}=\alpha+\beta-1, \alpha^{\prime} \leq \alpha$ and $\beta^{\prime} \leq \beta$. Since $\left|V\left(G^{\prime}\right)\right|=\alpha^{\prime}+\beta^{\prime} \leq 3 r-2$, $G^{\prime}$ contains a geodesic cycle $C_{2 r}$ or $C_{2 r+1}$ (see Theorem 2.2).

Further we distinguish five cases.

(1) $\alpha=2 r-1$

In this case $G^{\prime}=C_{2 r}$ and $\operatorname{es}\left(G^{\prime}\right)=\left(r^{2 r}\right)$, a contradiction.

(2) $\alpha=2 r-2$

In this case we have $\left|V\left(G^{\prime}\right)\right|=2 r+1$. If $G^{\prime}=C_{2 r+1}$ then $\operatorname{es}\left(G^{\prime}\right)=\left(r^{2 r+1}\right)$, 
a contradiction. If $G^{\prime}$ contains $C_{2 r}$ then (by Lemma 2.3a) $\operatorname{exc}_{G^{\prime}}\left(C_{2 r}\right) \leq 1$ and so $\alpha^{\prime}>\alpha$, a contradiction.

(3) $\alpha=2 r-2 i+1$

In this case we have $\left|V\left(G^{\prime}\right)\right|=2 r+i$. If $G^{\prime}$ contains $C_{2 r}$ then (by Lemma 4.3) $\operatorname{exc}_{G^{\prime}}\left(C_{2 r}\right) \leq i$ and so $\alpha^{\prime} \geq 2 r-i$. Since $i \geq 2$, we get $\alpha^{\prime}>$ $2 r-2 i+1=\alpha$, a contradiction. If $G^{\prime}$ contains $C_{2 r+1}$ then, by Lemma $2.3 \mathrm{~b}, \operatorname{exc}_{G^{\prime}}\left(C_{2 r+1}\right) \leq 2(i-1)$. Hence $\alpha^{\prime} \geq 2 r+1-2(i-1)=2 r-2 i+3>\alpha$, a contradiction.

(4) $\alpha=2 r-2 i$

In this case we have $\left|V\left(G^{\prime}\right)\right|=2 r+i+1$. If $G^{\prime}$ contains $C_{2 r}$ then $\operatorname{exc}_{G^{\prime}}\left(C_{2 r}\right) \leq i+1$ (see Lemma 4.3). Hence $\alpha^{\prime} \geq 2 r-(i+1)>2 r-2 i=\alpha$, a contradiction. If $G^{\prime}$ contains $C_{2 r+1}$ then $\operatorname{exc}_{G^{\prime}}\left(C_{2 r+1}\right) \leq 2 i$ (see Lemma $2.3 \mathrm{~b}$ ). Hence $\alpha^{\prime} \geq 2 r+1-2 i>2 r-2 i=\alpha$, and again we have a contradiction.

(5) $\frac{r+3}{2} \leq \alpha \leq \frac{2 r}{3}$

In this case $\left|V\left(G^{\prime}\right)\right|=4 r-2 \alpha+1$. If $G^{\prime}$ contains $C_{2 r}$ then $\operatorname{exc}_{G^{\prime}}\left(C_{2 r}\right) \leq$ $2 r-2 \alpha+1$ (see Lemma 4.3). Hence $\alpha^{\prime} \geq 2 r-(2 r-2 \alpha+1)=2 \alpha-1$. Since $\alpha \geq \frac{r+3}{2} \geq 6$, we get $\alpha>\alpha$, a contradiction. If $G^{\prime}$ contains $C_{2 r+1}$ then $\operatorname{exc}_{G^{\prime}}\left(C_{2 r+1}\right) \leq\left|V\left(G^{\prime}\right)\right|-(2 r+1)+\alpha^{\prime}=2 r-2 \alpha+\alpha^{\prime}$ (see Lemma 4.3). We get $\alpha^{\prime} \geq 2 r+1-\left(2 r-2 \alpha+\alpha^{\prime}\right)=2 \alpha-\alpha^{\prime}+1$ and so we have $2 \alpha^{\prime} \geq 2 \alpha+1$. Hence $\alpha^{\prime}>\alpha$, a contradiction.

c) We show that there is no other minimal eccentric sequence of type $r^{\alpha},(r+$ 1) ${ }^{\beta}$ for $r \geq 5$ and $\alpha \geq \frac{r+3}{2}$.

Since $r^{2 r-1},(r+1)^{2}$ is a minimal eccentric sequence, it follows that $r^{\alpha},(r+$ $1)^{\beta}$ for $\alpha \geq 2 r$ is not a minimal eccentric sequence (see Theorem 2.1). It is easy to check that for each $\alpha$ satisfying inequalities $\frac{r+3}{2} \leq \alpha \leq 2 r-1$ we already have a minimal eccentric sequence of type $r^{\alpha},(r+1)^{\beta}$. It is clear that if $\beta_{1} \neq \beta_{2}$ then at most one of the two sequences $r^{\alpha},(r+1)^{\beta_{1}}$ and $r^{\alpha},(r+1)^{\beta_{2}}$ is a minimal eccentric sequence.

If we take the parts a), b), c) into account, the proof is finished.

Remark 3.3. In most cases minimal eccentric sequences from Theorem 3.2 are realizable through many different graphs. An exception is for instance the sequence $r^{2 r-1},(r+1)^{2}$. Note that if a minimal eccentric sequence is realizable through a sun-graph then its rays can in general be deployed in different ways (see the proof of Theorem 3.4 in [7]). If a graph which realizes a minimal eccentric sequence from Theorem 3.2 has, for instance, a subgraph depicted in 


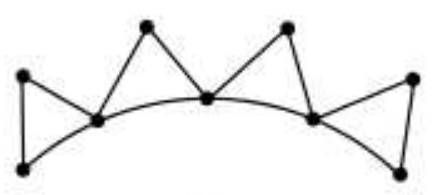

a)
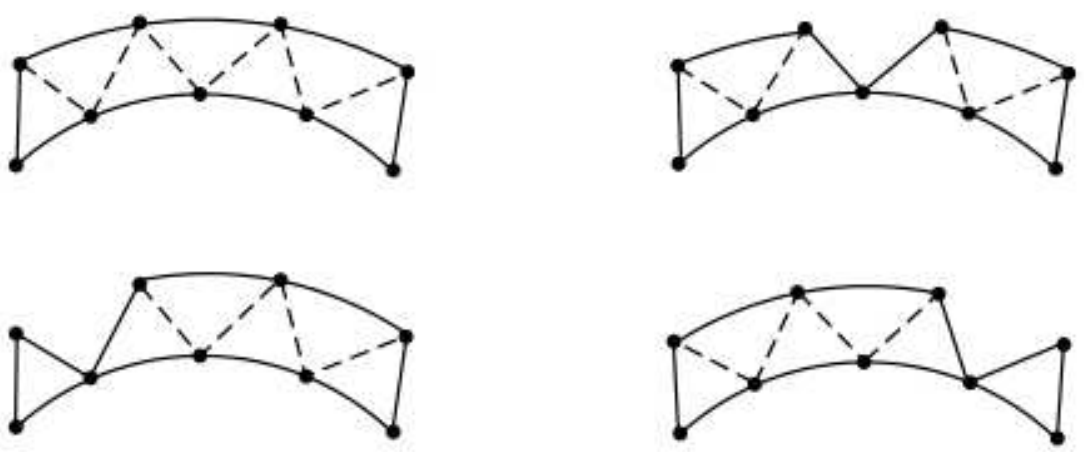

b)

Figure 3

Figure 3a, then this subgraph can be replaced by any of the graphs in Figure 3b containing an arbitrary subset of dashed edges.

Remark 3.4. It follows from Theorem 3.2 that the conjecture about minimal two-valued eccentric sequences given in [8] does not hold. All minimal eccentric sequences of type $r^{\alpha},(r+1)^{\beta}$ are known for $r \in\{1,2,3,4\}$ (see Theorems 2.5, 2.6 and 2.7). If $r \geq 5$ then all minimal eccentric sequences of type $r^{\alpha},(r+1)^{\beta}$ are known for $\alpha \geq \frac{r+3}{2}$ (Theorem 3.2).

Conjecture 3.5. All minimal eccentric sequences of type $r^{\alpha},(r+1)^{\beta}$ for $r \geq 3$ and $\alpha<\frac{r+3}{2}$ are

(i) $r^{\alpha},(r+1)^{4 r-3 \alpha+2}, \quad 2 \leq \alpha<\frac{r+3}{2}$,

(ii) $r,(r+1)^{4 r-2}$.

Remark 3.6. Note that Conjecture 3.5 holds for $r \in\{3,4\}$ (see Theorems 2.6 and 2.7 ).

Remark 3.7. Graphs realizing eccentric sequences from the case (i) of Conjecture 3.5 can be found using the procedure described in the proof of 


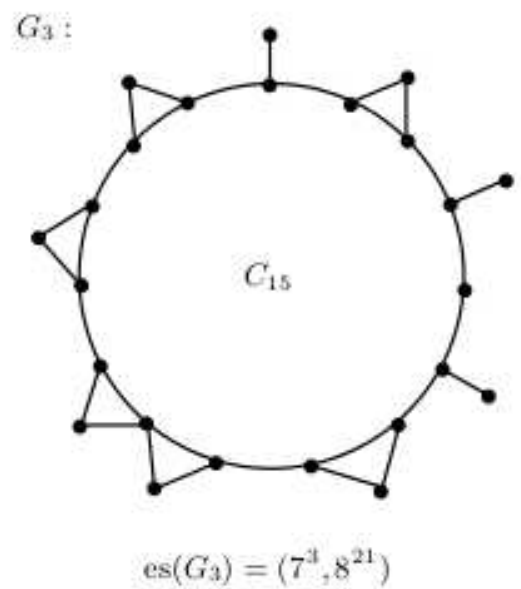

Figure 4

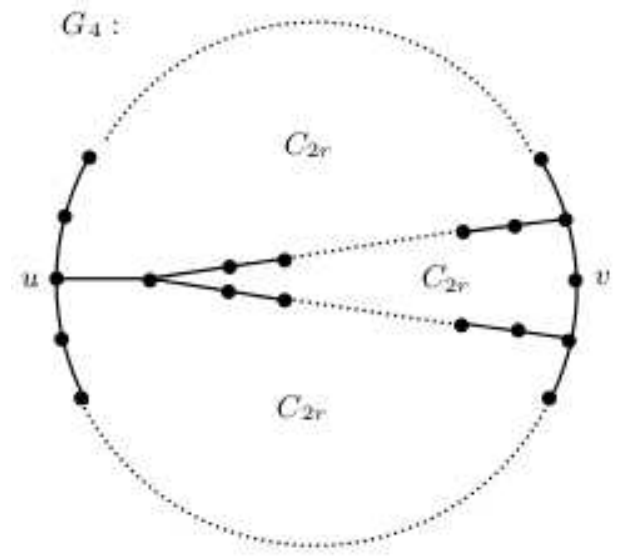

$d_{G_{4}}(u, v)=r+1$
$\operatorname{es}\left(G_{4}\right)=\left(r,(r+1)^{4 r-2}\right)$

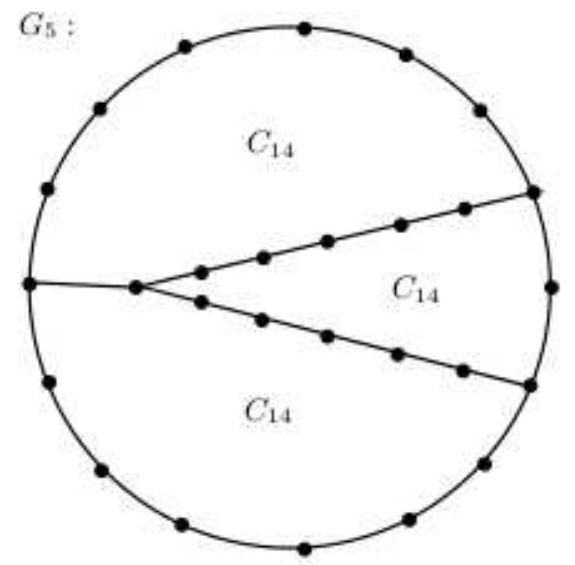

$\operatorname{es}\left(G_{5}\right)=\left(7,8^{20}\right)$

Figure 5

Theorem $3.2\left(\right.$ see the steps $\left.\left(1^{\circ}\right),\left(2^{\circ}\right)\right)$. For instance the graph $G_{3}$ with $\operatorname{es}\left(G_{3}\right)=$ $\left(7^{3}, 8^{21}\right)$ is shown in Figure 4.

The eccentric sequence $r,(r+1)^{4 r-2}$ (the case (ii) of Conjecture 3.5) is realizable through a graph $G_{4}$ in Figure 5 (e.g. the graph $G_{5}$ with $\operatorname{es}\left(G_{5}\right)=$ $\left(7,8^{26}\right)$ is shown in Figure 5, too). 


\section{Proofs of Auxiliary Results}

In this section we give proofs of results used in the previous section.

Lemma 4.1. The sequence $5^{4}, 6^{11}$ is a minimal eccentric sequence.

Proof. The sequence $5^{4}, 6^{11}$ is eccentric (see Figure 1). Now we are going to show that it is minimal.

Let $G$ be a graph for which es $(G)=\left(5^{\alpha}, 6^{\beta}\right), \alpha+\beta=14, \alpha \leq 4$ and $\beta \leq 11$. By Theorem 2.4c $G$ contains a cycle $C_{m}, m \geq 10$. We distinguish three cases.

(1) $m=11$

By Lemma $2.3 \mathrm{~b}$ we get $\operatorname{exc}_{G}\left(C_{11}\right) \leq 6$ and it implies $\alpha \geq 5$, a contradiction.

(2) $m=10$

By Lemma 2.3c we have $l=\left|\left\{v \in V(G) ; d\left(v, C_{10}\right)=1\right\}\right| \leq 2$. It is easy to see that the case $l=1$ cannot occur. If $l=2$ then $\operatorname{exc}_{G}\left(C_{10}\right) \leq 6$ (see Lemma 2.3c). It follows that $e_{G}(u)=6$ for every vertex $u \in V(G) \backslash V\left(C_{10}\right)$ and consequently $u$ is not a cut-vertex of $G$. Now it is easy to check that $\alpha \geq 6$, a contradiction.

(3) $m \in\{12,13,14\}$

According to the cases (1), (2) we can assume that $G$ contains neither $C_{10}$ nor $C_{11}$. Obviously, $C_{m}$ is not a geodesic cycle. Now it is easy to verify that $\operatorname{rad} G<5$, a contradiction.

Now consider graphs $H_{1}, H_{2}$ schematically depicted in Figure 6 with the properties $d_{C_{m}}\left(u_{1}, u_{2}\right)=r, d_{C_{m}}\left(v_{1}, v_{2}\right)+d_{C_{m}}\left(v_{2}, v_{3}\right)+d_{C_{m}}\left(v_{3}, v_{1}\right)=m$.

Lemma 4.2. Let $G$ be a graph with radius $r$ and with at most $3 r-2$ vertices. Let $C_{m}, m \in\{2 r, 2 r+1\}$, be a geodesic cycle of $G$. Then neither $H_{1}$ nor $\mathrm{H}_{2}$ is a subgraph of $G$.

Proof. a) Let $H_{1}$ be a subgraph of $G$ (note that $d_{C_{m}}\left(u_{1}, u_{2}\right)=r$ ). Since $C_{m}$ is a geodesic cycle, the graph $G$ has at least $3 r-1$ vertices, a contradiction.

b) Let $H_{2}$ be a subgraph of $G$ and $H_{1}$ be not a subgraph of $G$. We have $\left|V\left(H_{2}\right) \backslash V\left(C_{m}\right)\right|=x_{1}+x_{2}+x_{3}+1$ (see Figure 6). Since $C_{m}$ is a 

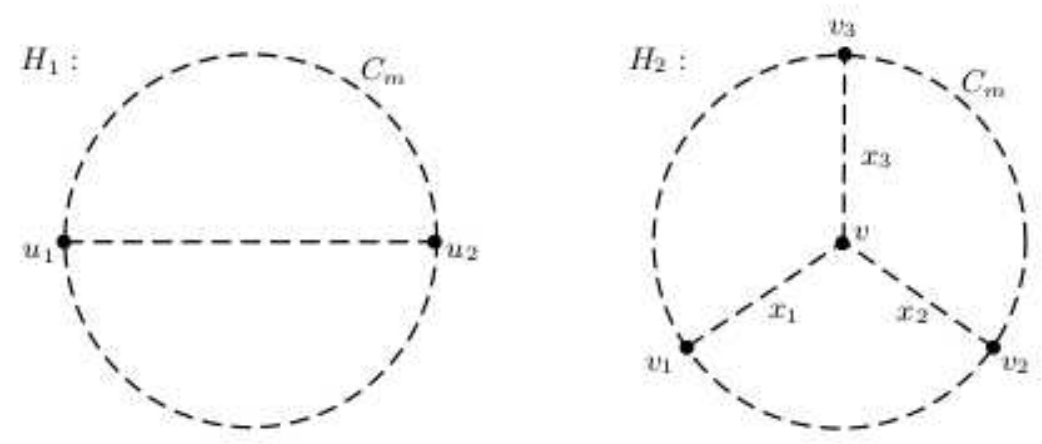

Figure 6

geodesic cycle, we have $\left(x_{1}+1+x_{2}\right)+\left(x_{2}+1+x_{3}\right)+\left(x_{3}+1+x_{1}\right) \geq m-3$. Hence $x_{1}+x_{2}+x_{3} \geq \frac{m}{2}-3$. If $m=2 r+1$ then $x_{1}+x_{2}+x_{3}>r-3$ and we get $|V(G)|>(2 r+1)+(r-3)+1=3 r-1$, a contradiction. If $m=2 r$ then $x_{1}+x_{2}+x_{3} \geq r-3$. If $x_{1}+x_{2}+x_{3}>r-3$ or $|V(G)|>\left|V\left(H_{2}\right)\right|$, then $|V(G)|>3 r-2$, a contradiction. So we can assume that $x_{1}+x_{2}+x_{3}=r-3$ and $\left|V\left(H_{2}\right)\right|=|V(G)|$. Now we have $r-1 \geq d_{C_{2 r}}\left(v_{i}, v_{j}\right)=d_{G}\left(v_{i}, v_{j}\right)=x_{i}+x_{j}+2$ for $i \neq j$. Hence $e_{G}(v) \leq r-1$, a contradiction.

Lemma 4.3. Let $G$ be a graph with es $(G)=\left(r^{\alpha},(r+1)^{\beta}\right), r \geq 3$ and $\alpha+\beta \leq 3 r-2$. Let $C_{m}, m \in\{2 r, 2 r+1\}$, be a geodesic cycle of $G$. Then

(i) $\operatorname{exc}_{G}\left(C_{2 r}\right) \leq|V(G)|-2 r$,

(ii) $\operatorname{exc}_{G}\left(C_{2 r+1}\right) \leq \min \{|V(G)|-(2 r+1)+\alpha, 2(|V(G)|-(2 r+1))\}$.

Proof. Consider a vertex $w \in V(G) \backslash V\left(C_{m}\right)$. Let $W$ be a component of $G-V\left(C_{m}\right)$ such that $w \in V(W)$. Further let $V_{1}=\left\{x \in V\left(C_{m}\right) ; d_{G}(x, W)=1\right\}$ and let $a=\max \left\{d_{G}(x, y) ; x, y \in V_{1}\right\}$ (note that $d_{C_{m}}(x, y)=d_{G}(x, y)$ for $x, y \in$ $\left.V\left(C_{m}\right)\right)$. Let $u, v \in V_{1}$ be vertices with $d_{G}(u, v)=a$. Then $a<r$ and for any vertex $x \in V_{1}$ it holds $d_{C_{m}}(u, x)+d_{C_{m}}(v, x)=a$ (see Lemma 4.2).

Let $u \underline{W} v$ denote a path such that all its vertices except $u, v$ belong to $V(W)$. Since $C_{m}$ is a geodesic cycle of $G$, the length of the considered path is at least $a$ and so $|V(W)| \geq a-1$. Let $a_{1}=\max \left\{d_{G}(u, x) ; x \in V(W)\right\}$ and $a_{2}=\max \left\{d_{G}(v, x) ; x \in V(W)\right\}$. Obviously, $a_{i} \geq a-1, i \in\{1,2\}$. Now we are going to show that $a_{i} \leq a+1$. Suppose, contrary to our claim, that there exists a vertex $x \in V(W)$ for which $d_{G}(u, x) \geq a+2$. Hence $d_{G}(v, x) \geq 2$ (since $\left.d_{G}(u, v)=a\right)$. Now it is easy to verify that there is a vertex $y \in V\left(C_{m}\right)$ for 


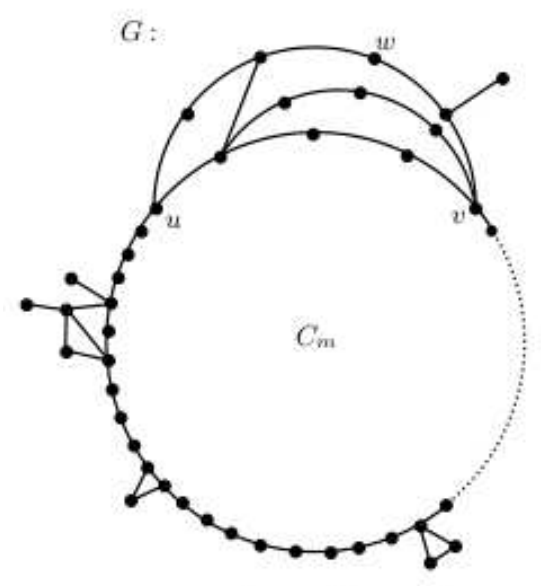

$a=4, a_{1}=5, \alpha_{2}=4$

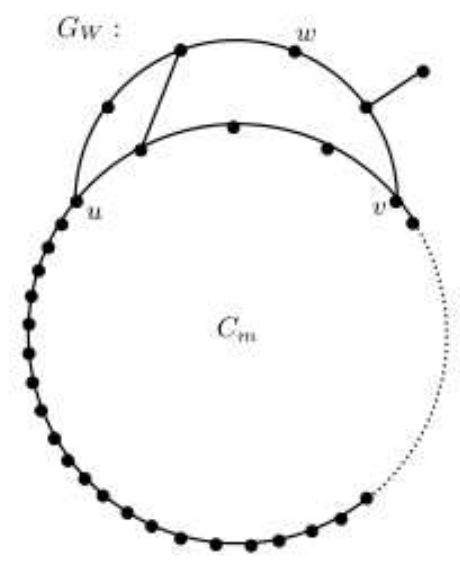

Figure 7

which $d_{G}(x, y) \geq r+2$, a contradiction. In what follows we can assume that $a-1 \leq a_{i} \leq a+1, i \in\{1,2\}$.

Let $G_{W}$ denote the subgraph of $G$ induced by $V\left(C_{m}\right) \cup V(W)$ (see Figure $7)$. Let $b$ be the length of some $u \underline{W} v$ path. Obviously, $b \geq a\left(C_{m}\right.$ is a geodesic cycle). Since $|V(G)| \leq 3 r-2$, we have $|V(W)| \leq r-2$. Let $V^{a}=\{x \in$ $\left.V\left(C_{m}\right) ; d_{C_{m}}(u, x)+d_{C_{m}}(x, v)=a\right\}$. Obviously, $\left|V^{a}\right|=a+1$. If $s \in V^{a}$ and $t \in V(W)$ then $d_{G_{W}}(s, t)=d_{G}(s, t) \leq\left\lfloor\frac{a+b}{2}\right\rfloor+((r-2)-(b-1)) \leq \frac{a+b}{2}+r-b-1=$ $\frac{a-b}{2}+r-1 \leq r-1$. Hence $e_{G_{W}}(s)=r$ for every vertex $s \in V^{a}$.

(i) Let $m=2 r$.

If $a=0$ then $u=v$ and $u$ is a cut-vertex of $G$. Hence $\operatorname{exc}_{G_{W}}\left(C_{2 r}\right)=1 \leq$ $|V(W)|$. So we can assume that $a>0$. Further we distinguish two cases.

(1) $a_{i} \in\{a-1, a\}, i \in\{1,2\}$

Obviously, in the cycle $C_{2 r}$ there exist at least $a+1+2(r-a)=$ $2 r-a+1$ vertices with the eccentricity $r$ in $G_{W}$. Hence $\operatorname{exc}_{G_{W}}\left(C_{2 r}\right) \leq$ $2 r-(2 r-a+1)=a-1 \leq|V(W)|$.

(2) $\max \left\{a_{1}, a_{2}\right\}=a+1$

It is clear that in the cycle $C_{2 r}$ there exist at least $(a+1)+2(r-$ $(a+1))=2 r-a-1$ vertices with eccentricity $r$ in $G_{W}$. Hence $\operatorname{exc}_{G_{W}}\left(C_{2 r}\right) \leq 2 r-(2 r-a-1)=a+1$. It is easy to verify (by considering the length of a shortest $u \underline{W} v$ path) that $|V(W)| \geq a+1$. 
From what has already been proved $\left(\operatorname{exc}_{G_{W}}\left(C_{2 r}\right) \leq|V(W)|\right)$, it easily follows that the proof of the case (i) is finished.

(ii) Let $m=2 r+1$.

If $a=0$ then $u=v$ and $u$ is a cut-vertex of $G$. Hence $e_{G}(u)=r$ and so $\operatorname{exc}_{G_{W}}\left(C_{2 r+1}\right)=2 \leq|V(W)|+1$. Let $a>0$. If $|V(W)|=a-1$ then $\operatorname{exc}_{G_{W}}\left(C_{2 r+1}\right)=0<|V(W)|$. So we can assume that $|V(W)| \geq a>0$. We distinguisch three cases.

(1) $a_{i} \in\{a-1, a\}, i \in\{1,2\}$

In the cycle $C_{2 r+1}$ there exist at least $a+1+2(r-a)=2 r-a+1$ vertices with eccentricity $r$ in $G_{W}$. It implies $\operatorname{exc}_{G}\left(C_{2 r+1}\right) \leq 2 r+$ $1-(2 r-a+1)=a \leq|V(W)|$.

(2) $a_{i}=a, a_{j}=a+1,\{i, j\}=\{1,2\}$

We have $\operatorname{exc}_{G_{W}}\left(C_{2 r+1}\right) \leq(2 r+1)-((a+1)+(r-a)+(r-(a+1)))=$ $a+1$. It is easy to verify (by considering the length of a shortest $u \underline{W} v$ path) that $|V(W)| \geq a+1$. We get $\operatorname{exc}_{G_{W}}\left(C_{2 r+1}\right) \leq|V(W)|$.

(3) $a_{1}=a_{2}=a+1$

Firstly we show that $e_{G}(u)=e_{G}(v)=r$. Suppose, contrary to our claim, that e.g. $e_{G}(u)=r+1$. Since we know that $e_{G_{W}}(v)=$ $e_{G_{W}}(u)=r$, there exists a vertex $x \in V(G) \backslash V(W)$ such that $d_{G}(u, x)=r+1$. Obviously, $d_{G}(v, x) \geq r+1-a$. Since $a_{2}=a+1$, there exists a vertex $y \in V(W)$ such that $d_{G}(v, y)=a+1$. Now it is easy to verify that $d_{G}(x, y) \geq r+2$, a contradition.

Clearly, $|V(W)| \geq a+1$ and $\operatorname{exc}_{G_{W}}\left(C_{2 r+1}\right) \leq(2 r+1)-((a+$ 1) $+2(r-a-1))=a+2$. Finally, in the case (3), we have $\operatorname{exc}_{G_{W}}\left(C_{2 r+1}\right) \leq|V(W)|+1$ and $e_{G}(u)=e_{G}(v)=r$.

If we take the possibility $a=0$ and the cases (1), (2), (3) into account we get $\operatorname{exc}_{G}\left(C_{2 r+1}\right) \leq|V(G)|-(2 r+1)+\alpha$. According to Lemma 2.3b we have $\operatorname{exc}_{G}\left(C_{2 r+1}\right) \leq 2(|V(G)|-(2 r+1))$ and so the proof is finished.

\section{Acknowledgments}

The authors were supported by the Slovak Grant Agency under the grant number VEGA 1/1085/11 


\section{References}

[1] F. Buckley, Eccentric sequences, eccentric sets, and graph centrality, Graph Theory Notes N. Y. 40 (2001), 18-22.

[2] F. Buckley and F. Harary, Distance in Graphs, Addison-Wesley Publishing Company, Redwood City, CA, 1990.

[3] F. Buckley and F. Harary, Unsolved problems on distance in graphs, Electron. Notes Discerte Math., Vol. 11 (2002), 89-97.

[4] A. A. Dobrynin, Degeneracy of some matrix graph invariants, J. Math. Chem. 14 (1993), no. 2, 175-184.

[5] D. Ferrero and F. Harary, On eccentricity sequences of connected graphs, AKCE Int. J. Graphs Comb. 6 (2009), no. 3, 401-408.

[6] A. Haviar, P. Hrnčiar and G. Monoszová, Minimal eccentric sequences with least eccentricity three, Acta Univ. M. Belii, Ser. Math. no. 5 (1997), 27-50.

[7] A. Haviar, P. Hrnčiar and G. Monoszová, Eccentric sequences and cycles in graphs, Acta Univ. M. Belii, Ser. Math. no 11 (2004), 7-25.

[8] P. Hrnčiar and G. Monoszová, Minimal eccentric sequences with two values, Acta Univ. M. Belii, Ser. Math. no 12 (2005), 43-65.

[9] W.G. Kropatsch, A. Ion, Y. Haxhimusa and T. Flanitzer, The eccentricity transform (of a digital shape), Discrete Geometry for Computer Imagery, 437-448, Lecture Notes in Comput. Sci., 4245, Springer, Berlin, 2006.

[10] L. Lesniak, Eccentric sequences in graphs, Period. Math. Hungar 6 (1975), $287-293$.

[11] R. Nandakumar, On some eccentricity properties of graphs, Ph.D. thesis, Indian Institute of Technology, Madras (1986).

[12] P. A. Ostrand, Graphs with specified radius and diameter, Discrete Math. 4 (1973), 71-75.

[13] M. Saheli and A. R. Ashrafi The eccentric connectivity index of armchair polyhex nanotubes, Macedonian Journal of Chemistry and Chemical Engineering, Vol 29, no. 1 (2010), 71-75. 